\title{
Sums of Products Involving Power Sums of $\varphi(n)$ Integers
}

\author{
Jitender Singh \\ Department of Mathematics, Guru Nanak Dev University, Amritsar 143005, India \\ Correspondence should be addressed to Jitender Singh; sonumaths@gmail.com
}

Received 12 November 2013; Accepted 22 January 2014; Published 27 February 2014

Academic Editor: Junesang Choi

Copyright (C) 2014 Jitender Singh. This is an open access article distributed under the Creative Commons Attribution License, which permits unrestricted use, distribution, and reproduction in any medium, provided the original work is properly cited.

A sequence of rational numbers as a generalization of the sequence of Bernoulli numbers is introduced. Sums of products involving the terms of this generalized sequence are then obtained using an application of Faà di Bruno's formula. These sums of products are analogous to the higher order Bernoulli numbers and are used to develop the closed form expressions for the sums of products involving the power sums $\Psi_{k}(x, n):=\sum_{d \mid n} \mu(d) d^{k} S_{k}(x / d), n \in \mathbb{Z}^{+}$which are defined via the Möbius function $\mu$ and the usual power sum $S_{k}(x)$ of a real or complex variable $x$. The power sum $S_{k}(x)$ is expressible in terms of the well-known Bernoulli polynomials by $S_{k}(x):=\left(B_{k+1}(x+1)-B_{k+1}(1)\right) /(k+1)$.

\section{Introduction}

Singh [1] introduced the power sum $\Psi_{k}(x, n)$ of real or complex variable $x$ and positive integer $n$ defined by the generating function

$$
\sum_{d \mid n} \mu(d) \frac{e^{(1+(x / d)) t d}-e^{t d}}{e^{t d}-1}=\sum_{k=0}^{\infty} \Psi_{k}(x, n) \frac{t^{k}}{k !}
$$

from which he derived the following closed form formula for these power sums:

$$
\Psi_{k}(x, n)=\frac{1}{k+1} \sum_{m=0}^{[k / 2]}\left(\begin{array}{c}
k+1 \\
2 m
\end{array}\right) B_{2 m} x^{k+1-2 m} \prod_{p \mid n}\left(1-p^{2 m-1}\right)
$$

for all $k=0,1, \ldots$ where $B_{m}$ are the Bernoulli numbers and $p$ runs over all prime divisors of $n$. In particular, $\Psi_{k}(n, n)$ gives the sum of $k$ th power of those positive integers which are less than $n$ and relatively prime to $n$. We will call $\Psi_{k}(x, n)$ as Möbius-Bernoulli power sums. Present work is aimed at describing sums of products of the power sums $\Psi_{k}(x, n)$ via introducing yet another sequence of rational numbers which we will call as the sequence of Möbius-Bernoulli numbers. The rational sequence $\left\{B_{k}\right\}$ that appears in (2) is defined via the generating function $t /\left(e^{t}-1\right)=\sum_{k=0}^{\infty} B_{k}\left(t^{k} / k !\right),|t|<2 \pi$, and was known to Faulhaber and Bernoulli. Many explicit formulas for the Bernoulli numbers are also well known in the literature. One such formula is as follows [2]:

$$
B_{k}=\sum_{m=1}^{k} \frac{1}{m+1} \sum_{n=1}^{m}(-1)^{n}\left(\begin{array}{c}
m \\
n
\end{array}\right) n^{k}, \quad k=0,1, \ldots
$$

The rest of the paper is organized as follows. Möbius Bernoulli numbers are introduced in Section 2 and their sums of products are discussed via Faà di Bruno's formula. In Section 3 sums of products of power sums of $\varphi(n)$ integers are obtained in closed form using sums of products of Möbius Bernoulli numbers.

\section{Möbius-Bernoulli Numbers}

Definition 1. We define Möbius-Bernoulli numbers $M_{k}(n)$, $k=0,1, \ldots$, via the generating function

$$
\sum_{d \mid n} \frac{t \mu(d)}{e^{t d}-1}=\sum_{k=0}^{\infty} M_{k}(n) \frac{t^{k}}{k !}, \quad|t|<\frac{2 \pi}{n}, \quad \forall n \in \mathbb{Z}^{+} .
$$

We immediately notice from (4) that the Möbius-Bernoulli numbers are given by

$$
\begin{array}{r}
M_{k}(1)=B_{k} ; \quad M_{k}(n)=B_{k} \prod_{p \mid n}\left(1-p^{k-1}\right), \\
\forall n \geq 2, k=0,1, \ldots .
\end{array}
$$


Note that, for a fixed $k$, the Möbius Bernoulli number $M_{k}(n)$ is a multiplicative function of $n$. Singh [1] has obtained the following identity relating the function $\Psi_{k}(x, n)$ to the Möbius-Bernoulli numbers. $(d / d x) \Psi_{k}(x, n)=k \Psi_{k-1}(x, n)+$ $(-1)^{k} M_{k}(n)$, from which we observe that $\Psi_{k}(x, 1)=S_{k}(x)$, $\Psi_{0}(x, n)=\varphi(n)$, and $\Psi_{k}(0, n)=0$ for all $k=0,1, \ldots$, where $\varphi(n)$ is Euler's totient. Use of Möbius Bernoulli numbers is inherent in studies recently done by Alkan [3] on averages of Ramanujan sums which are defined for any complex number $z$ and integer $k$ by $c_{k}(z):=\sum_{q \in \Phi(k)} e^{2 \pi \imath q z / k}$, where $\Phi(k)=$ $\{q \mid 1 \leq q \leq k, \operatorname{gcd}(q, k)=1\}$. Möbius Bernoulli numbers are also related to Jordan's totient (a generalization of Euler's totient) $J_{k}(n):=n^{k} \prod_{p \mid n}\left(1-p^{-k}\right)$ by $n^{2 k-1} M_{2 k}(n)=$ $-B_{2 k} c(n)^{2 k-1} J_{2 k-1}(n)$, where $c(n)$ is the square free part of $n$. The notion of Bernoulli polynomials generalizes to Möbius Bernoulli polynomials which we define next.

Definition 2. We say $M_{k}(x, n)$ is the $k$ th Möbius Bernoulli polynomial defined by the generating function

$$
\begin{array}{r}
\sum_{d \mid n} \mu(d) \frac{t e^{x t}}{e^{t d}-1}=\sum_{k=0}^{\infty} M_{k}(x, n) \frac{t^{k}}{k !}, \quad|t|<\frac{2 \pi}{n}, \\
x \in \mathbb{C}, n \in \mathbb{Z}^{+}
\end{array}
$$

From (6), we see that $M_{0}(x, n)=M_{0}(n), M_{k}(0, n)=$ $M_{k}(n)$, and $(d / d x) M_{k}(x, n)=k M_{k-1}(x, n)$ for all $k \in \mathbb{Z}^{+}$ and that $M_{k}(x, 1)=B_{k}(x)$, where $B_{k}(x)$ is the $k$ th Bernoulli polynomial. It also follows from (6) that Möbius Bernoulli polynomials are given by

$$
M_{k}(x, n)=\sum_{d \mid n} \mu(d) B_{k}\left(\frac{x}{d}\right) d^{k-1}=\sum_{m=0}^{k}\left(\begin{array}{c}
k \\
m
\end{array}\right) M_{k-m}(n) x^{m} .
$$

If $\Psi_{k}(x, n)$ is as before, we have $\Psi_{0}(x, n)=M_{1}(x, n)-$ $M_{1}(0, n)=x \varphi(n) / n$ and

$$
\begin{array}{r}
\Psi_{k}(x, n)=x^{k} \sum_{d \mid n} \mu(d)+\frac{M_{k+1}(x, n)-M_{k+1}(0, n)}{k+1}, \\
k=1,2, \ldots .
\end{array}
$$

Definition 3. Let $n, N$ be positive integers, and let $k$ be a nonnegative integer. Define higher order Möbius-Bernoulli numbers by

$$
M_{k}^{N}(n):=\sum_{\sum_{i=1}^{N} k_{i}=k}\left(\begin{array}{c}
k \\
k_{1}, \ldots, k_{N}
\end{array}\right) M_{k_{1}}(n) \cdots M_{k_{N}}(n),
$$

which are described by the generating function

$$
H(t)=\left(\sum_{d \mid n} \frac{t \mu(d)}{e^{t d}-1}\right)^{N}=\sum_{k=0}^{\infty} M_{k}^{N}(n) \frac{t^{k}}{k !}, \quad|t|<\frac{2 \pi}{n} .
$$

Note that $M_{k}^{N}(1)$ is the higher order Bernoulli number [4]. Also, $M_{k}^{1}(n)=M_{k}(n)$ for all $k=0,1, \ldots$. Some of the first few higher order Möbius-Bernoulli numbers are given by the following:

$$
\begin{gathered}
M_{0}^{N}(n)=(\varphi(n))^{N}, \\
M_{2}^{N}(n)=N(\varphi(n))^{N-1} M_{2}(n), \\
M_{4}^{N}(n) \\
=N(\varphi(n))^{N-2}\left\{3(N-1)\left(M_{2}(n)\right)^{2}+\varphi(n) M_{4}(n)\right\}, \\
M_{6}^{N}(n)=N(\varphi(n))^{N-3} \\
\times\left\{15(N-1)(N-2)\left(M_{2}(n)\right)^{2}\right. \\
+15(N-1) \varphi(n) M_{2}(n) M_{4}(n) \\
\left.+\varphi(n)^{2} M_{6}(n)\right\} \text { etc. }
\end{gathered}
$$

Note that $M_{k}^{N}(n)=\lim _{t \rightarrow 0}\left(d^{k} H(t) / d t^{k}\right)$. In this regard, a formula for the higher order Möbius-Bernoulli numbers can be obtained from the following version of the well-known Faà di Bruno's formula [5].

Lemma 4. Let $N$ be a positive integer, and let $f: \mathbb{R} \rightarrow \mathbb{R}$ be a function of class $C_{k}, k \geq 1$. Then

$$
\begin{aligned}
& D^{k}(f(x))^{N} \\
& =N ! \sum_{j=1}^{k} \frac{(f(x))^{N-j}}{(N-j) !} \sum_{\sum_{i=1}^{j} k_{i}=k}\left(\begin{array}{c}
k \\
k_{1}, \ldots, k_{j}
\end{array}\right) \\
& \\
& \quad \times \frac{D^{k_{1}} f(x) \cdots D^{k_{j}} f(x)}{\lambda\left(k_{1}\right) ! \cdots \lambda\left(k_{j}\right) !},
\end{aligned}
$$

where $D^{k}=d^{k} / d x^{k}, k=1,2, \ldots ; \lambda\left(k_{i}\right)$ is the multiplicity of occurrence of $k_{i}$ in the partition $\left\{k_{1}, \ldots, k_{j}\right\}$ of $n$ of length $j$; and $\lambda\left(k_{i}\right)$ ! contributes only once in the above product.

Proof. We use induction on $k$ in proving the result. For $k=1$, we see that $j=1$ in the RHS of (12) and it reduces to $N(f(x))^{N-1} D^{1}(f(x))=D^{1}\left(f(x)^{N}\right)$. This proves that the result is true for $k=1$. Let us assume that formula (12) holds for all positive integers $\leq k$. Now assume that $f$ is of class $C_{k+1}$ and consider

$$
\begin{aligned}
D^{k+1}\left((f(x))^{N}\right) \\
=N ! \sum_{j=1}^{k-1} \frac{(f(x))^{N-(j+1)}}{(N-(j+1)) !} \\
\quad \times \sum_{\sum_{i=1}^{j} k_{i}=k}\left(\begin{array}{c}
k \\
k_{1}, \ldots, k_{j}
\end{array}\right)
\end{aligned}
$$




$$
\begin{aligned}
& \times \frac{D^{1} f(x) D^{k_{1}} f(x) \cdots D^{k_{j}} f(x)}{\lambda\left(k_{1}\right) ! \cdots \lambda\left(k_{j}\right) !} \\
& +N ! \frac{(f(x))^{N-k-1}}{(N-k-1) !}(D f(x))^{k+1} \\
& +N ! \sum_{j=1}^{k} \frac{(f(x))^{N-j}}{(N-j) !} \\
& \quad \times \sum_{\sum_{i=1}^{j} k_{i}=k}\left(k_{1}, \ldots, k_{j}\right) \\
& \quad \times \frac{D\left(D^{k_{1}} f(x) \cdots D^{k_{j}} f(x)\right)}{\lambda\left(k_{1}\right) ! \cdots \lambda\left(k_{j}\right) !} .
\end{aligned}
$$

At this point, observe that any partition $\pi^{\prime}$ of $k+1$ can be obtained from a partition $\pi$ of $k$ by adjoining 1 , and let us denote the set of all such partitions of $k+1$ by $S$. Denote by $T$ the set of remaining all partitions of $k+1$ where each $\pi^{\prime}$ is obtained simply by adding 1 to exactly one member of $\pi$. In each of these cases one has $k+1$ choices of doing so for a fixed $\pi$. In the former case for each $\pi^{\prime} \in S,\left|\pi^{\prime}\right|=|\pi|+1$ which happens in the first summation above in (13) which reduces to the following:

$$
\begin{aligned}
(k+1) N ! \sum_{j=1}^{k} \frac{(f(x))^{N-j}}{(N-j) !} & \\
& \times \sum_{\left\{k_{1}^{\prime}, \ldots, k_{j+1}^{\prime}\right\} \in S}\left(\begin{array}{c}
k \\
k_{1}^{\prime}, \ldots, k_{j+1}^{\prime}
\end{array}\right) \\
& \times \frac{D^{k_{1}^{\prime}} f(x) \cdots D^{k_{j+1}^{\prime}} f(x)}{\lambda\left(k_{1}^{\prime}\right) ! \cdots \lambda\left(k_{j}^{\prime}\right) !} .
\end{aligned}
$$

In the latter case for each $\pi^{\prime} \in T,\left|\pi^{\prime}\right|=|\pi|$, and the terms after first summation in (13) reduce to

$$
\begin{aligned}
(k+1) N ! \sum_{j=1}^{k+1} \frac{(f(x))^{N-j}}{(N-j) !} & \\
& \times \sum_{\left\{k_{1}^{\prime}, \ldots, k_{j+1}^{\prime}\right\} \in T}\left(\begin{array}{c}
k \\
k_{1}^{\prime}, \ldots, k_{j+1}^{\prime}
\end{array}\right) \\
& \times \frac{D^{k_{1}^{\prime}} f(x) \cdots D^{k_{j+1}^{\prime}} f(x)}{\lambda\left(k_{1}^{\prime}\right) ! \cdots \lambda\left(k_{j}^{\prime}\right) !},
\end{aligned}
$$

where the term $N !\left((f(x))^{N-k-1} /(N-k-1) !\right)(D f(x))^{k+1}$ corresponds to $j=k+1$. The result follows by substituting (14) and (15) into (13). This completes the final step of induction.
Theorem 5. For each positive integers $N$ and $n$, the higher order Möbius-Bernoulli numbers are given by

$$
\begin{aligned}
M_{k}^{N}(n)=N ! \sum_{j=1}^{k} & \frac{(\varphi(n))^{N-j}}{(N-j) !} \\
& \times \sum_{\sum_{i=1}^{j} k_{i}=k}\left(\begin{array}{c}
k \\
k_{1}, \ldots, k_{j}
\end{array}\right) \\
& \times \frac{M_{k_{1}}(n) \cdots M_{k_{j}}(n)}{\lambda\left(k_{1}\right) ! \cdots \lambda\left(k_{j}\right) !} .
\end{aligned}
$$

Proof. First note from definition that $M_{k}^{N}(n)=\lim _{t \rightarrow 0} D^{k}$ $(H(0))$, the result follows at once by applying Lemma 4 to the function $f(t)=\sum_{d \mid n}\left(t \mu(d) /\left(e^{t d}-1\right)\right), 0<t<2 \pi / n$, and then taking limit $t \rightarrow 0$ throughout and using $\varphi(n)=$ $n \sum_{d \mid n}(\mu(d) / d)$ therein.

Proposition 6. $M_{2 k-1}^{N}(n)=0$ for all positive integers $k$ and $N$ and $n>1$.

Proof. Observe from (10) that, for a positive integer $N$, the following holds:

$$
\begin{aligned}
H(-t) & =\left(t \sum_{d \mid n} \mu(d)+\sum_{d \mid n} \frac{t \mu(d)}{e^{t d}-1}\right)^{N} \\
& =\left(t \delta_{1 n}+\sum_{d \mid n} \frac{t \mu(d)}{e^{t d}-1}\right)^{N} \\
& =H(t)
\end{aligned}
$$

for all $n>1$ where the arithmetic function $\sum_{d \mid n} \mu(d)=\delta_{1 n}$ is the Kronecker delta. We have proved that $H$ is an even function of $t$ for $n>1$. Thus the coefficient of $t^{2 k-1}$ in the RHS of (10) (which is precisely $M_{2 k-1}^{N}(n)$ ) vanishes for each $k=1,2, \ldots$.

Remark 7. If we extend the definition of higher MöbiusBernoulli numbers to complex $N \neq 0$, the formula (18) for $M_{k}^{N}(n)$ is still valid just on replacing $N ! /(N-j)$ ! by $N(N-$ 1) $\cdots(N-j+1)$ in it. In this regard we note that $M_{2 k-1}^{N}(n>$ 1) $=0$ holds for all $k=1,2, \ldots$ and $N \in \mathbb{C}$.

In view of the Theorem 5 and the Proposition 6, we have for all positive integers $n>1$ and $k=1,2, \ldots$,

$$
\begin{aligned}
M_{2 k}^{N}(n)=N ! \sum_{j=1}^{k} & \frac{(\varphi(n))^{N-2 j}}{(N-2 j) !} \\
& \times \sum_{\sum_{i=1}^{j} k_{i}=k}\left(\begin{array}{c}
2 k \\
2 k_{1}, \ldots, 2 k_{j}
\end{array}\right) \\
& \times \frac{M_{2 k_{1}}(n) \cdots M_{2 k_{j}}(n)}{\lambda\left(2 k_{1}\right) ! \cdots \lambda\left(2 k_{j}\right) !} .
\end{aligned}
$$


As an example, let $k=4$. There are five partitions of 4 which are given by $4_{1}, 3_{1} 1_{1}, 2_{2}, 2_{1} 1_{2}$, and $1_{4}$, and therefore from (18), we obtain

$$
\begin{aligned}
M_{8}^{N}(n) & \\
=N ! & \left\{\frac{\varphi(n)^{N-2}}{(N-2) !} M_{8}(n)+\frac{\varphi(n)^{N-4}}{(N-4) !}\right. \\
& \times\left(\frac{8 !}{6 ! 2 !} M_{6}(n) M_{2}(n)+\frac{8 !}{4 ! 4 !} \frac{\left(M_{4}(n)\right)^{2}}{2 !}\right) \\
& +\frac{\varphi(n)^{N-6}}{(N-6) !}\left(\frac{8 !}{4 ! 2 ! 2 !} M_{4}(n) \frac{\left(M_{2}(n)\right)^{2}}{2 !}\right) \\
& \left.+\frac{\varphi(n)^{N-8}}{(N-8) !} \frac{8 !}{2 ! 2 ! 2 ! 2 !} \frac{\left(M_{2}(n)\right)^{4}}{4 !}\right\} \\
=N ! & \left\{\frac{\varphi(n)^{N-2}}{(N-2) !} M_{8}(n)+\frac{\varphi(n)^{N-4}}{(N-4) !}\right. \\
& \times\left(28 M_{6}(n) M_{2}(n)+35\left(M_{4}(n)\right)^{2}\right) \\
& +\frac{\varphi(n)^{N-6}}{(N-6) !}\left(210 M_{4}(n)\left(M_{2}(n)\right)^{2}\right) \\
& \left.+\frac{\varphi(n)^{N-8}}{(N-8) !} 105\left(M_{2}(n)\right)^{4}\right\} .
\end{aligned}
$$

Remark 8. The formula (18) is not suitable for explicit evaluation of $M_{k}^{N}$ for large $k$. Because number of partitions of $k$ increases at a faster rate than $k$. For example the number of partitions of 10 is 42 , which is the number of terms in the expression for $M_{20}^{N}$. In this regard, it will be good to see a formula for the higher order Möbius Bernoulli numbers which can describe them better than the one we have given above.

Remark 9. If $n=p^{s}$ for some positive integer $s$ and prime $p$, then the simplest possible formula (18) for the higher order Möbius-Bernoulli numbers can be found as follows:

$$
\begin{aligned}
\left.H(t)\right|_{n=p^{s}} & =\left(\frac{t}{e^{t}-1}-\frac{t}{e^{t p}-1}\right)^{N} \\
& =\sum_{m=0}^{N}\left(\begin{array}{l}
N \\
m
\end{array}\right)\left(\frac{t}{e^{t}-1}\right)^{m}\left(\frac{t p}{e^{t p}-1}\right)^{N-m}(-p)^{m-N} .
\end{aligned}
$$

Therefore

$$
\begin{aligned}
M_{k}^{N}\left(p^{s}\right) & =\lim _{t \rightarrow 0} \frac{d^{k} H(t)}{d t^{k}} \\
& =\sum_{m=0}^{N}\left(\begin{array}{l}
N \\
m
\end{array}\right)(-p)^{m-N} \sum_{j=0}^{k}\left(\begin{array}{c}
k \\
j
\end{array}\right) B_{j}^{m} B_{k-j}^{N-m} p^{k-j},
\end{aligned}
$$

where we have utilized the Leibniz product rule for higher order derivatives and $B_{j}^{m}$ is the higher order Bernoulli number given by (see for more details Srivastava and Todorov [4])

$$
\begin{aligned}
B_{j}^{m}=\sum_{\ell=0}^{j}\left(\begin{array}{c}
j+m \\
j-\ell
\end{array}\right)\left(\begin{array}{c}
j+m-1 \\
\ell
\end{array}\right) & \\
& \times \frac{j !}{(j+\ell) !} \sum_{h=0}^{\ell}(-1)^{h}\left(\begin{array}{l}
\ell \\
h
\end{array}\right) h^{j+\ell} .
\end{aligned}
$$

Similarly, if we take $n=p_{1}^{s_{1}} p_{2}^{s_{2}}$ for some positive integers $s_{1}$, $s_{2}$ and distinct primes $p_{1}, p_{2}$, then

$$
\begin{aligned}
& \left.H(t)\right|_{n=p_{1}^{s_{1}} p_{2}^{s_{2}}} \\
& =\left(\frac{t}{e^{t}-1}-\frac{t}{e^{t p_{1}}-1}-\frac{t}{e^{t p_{2}}-1}+\frac{t}{e^{t p_{1} p_{2}}-1}\right)^{N} \\
& =\sum_{\sum_{i=0}^{3} m_{i}=N}\left(\begin{array}{c}
N \\
m_{0}, \ldots, m_{3}
\end{array}\right)\left(\frac{t}{e^{t}-1}\right)^{m_{0}} \\
& \quad \times\left(\frac{t p_{1}}{e^{t p_{1}}-1}\right)^{m_{1}}\left(\frac{t p_{2}}{e^{t p_{2}}-1}\right)^{m_{2}} \\
& \quad \times\left(\frac{t p_{1} p_{2}}{e^{t p_{1} p_{2}}-1}\right)^{m_{3}}\left(-p_{1}\right)^{-m_{1}}\left(-p_{2}\right)^{-m_{2}}\left(p_{1} p_{2}\right)^{-m_{3}}
\end{aligned}
$$

which gives

$$
\begin{gathered}
M_{k}^{N}\left(p_{1}^{s_{1}} p_{2}^{s_{2}}\right) \\
=\sum_{\sum_{i=0}^{3} m_{i}=N}\left(\begin{array}{c}
N \\
m_{0}, \cdots, m_{3}
\end{array}\right) \lim _{t \rightarrow 0} D^{k} \\
\times\left\{\left(\frac{t}{e^{t}-1}\right)^{m_{0}}\left(\frac{t p_{1}}{e^{t p_{1}}-1}\right)^{m_{1}}\right. \\
\left.\times\left(\frac{t p_{2}}{e^{t p_{2}}-1}\right)^{m_{2}}\left(\frac{t p_{1} p_{2}}{e^{t p_{1} p_{2}-1}}\right)^{m_{3}}\right\} \\
\times\left(-p_{1}\right)^{-m_{1}}\left(-p_{2}\right)^{-m_{2}}\left(p_{1} p_{2}\right)^{-m_{3}} \\
=\sum_{\sum_{i=0}^{3} m_{i}=N}\left(\begin{array}{c}
N \\
m_{0}, \cdots, m_{3}
\end{array}\right) \\
\times \sum_{\sum_{j=0}^{3} k_{i}=k}\left(\begin{array}{l}
k \\
k_{0}, \cdots, k_{3}
\end{array}\right) \\
\times B_{k_{0}}^{m_{0}} B_{k_{1}}^{m_{1}} B_{k_{2}}^{m_{2}} B_{k_{3}}^{m_{3}} p_{1}^{k_{1}-m_{1}} p_{2}^{k_{2}-m_{2}} \\
\times\left(p_{1} p_{2}\right)^{k_{3}-m_{3}}(-1)^{m_{1}+m_{2}} .
\end{gathered}
$$

These formulas involve products of higher order Bernoulli numbers. So in general, the formulas for $M_{k}^{N}(n)$ involve sums containing product of several higher order Bernoulli 
numbers and such a formula in the above sense would be complicated and will take the following form:

$$
\begin{aligned}
& M_{k}^{N}\left(\prod_{\alpha=1}^{\beta} p_{\alpha}^{e_{\alpha}}\right) \\
& =\frac{N ! k !}{(N+k) !} \\
& \quad \times \sum_{\sum_{i=0}^{2^{\beta}-1} m_{i}=N} \sum_{\sum_{j=0}^{2^{\beta}-1} k_{i}=k}\left(\begin{array}{c}
m_{0}, \ldots, m_{2^{\beta}-1}, k_{0}, \ldots, k_{2^{\beta}-1}
\end{array}\right) \\
& \quad \times B_{k_{0}}^{m_{0}} B_{k_{1}}^{m_{1}} \ldots B_{k_{2} \beta_{-1}}^{m_{\beta_{-1}}} \chi \\
& \quad \times\left(k_{1}, \ldots, k_{2^{\beta}-1}, m_{1}, \ldots, m_{2^{\beta}-1}\right),
\end{aligned}
$$

where

$$
\begin{gathered}
\chi\left(k_{1}, \ldots, k_{2^{\beta}-1}, m_{1}, \ldots, m_{2^{\beta}-1}\right) \\
=\prod_{s_{0}=1}^{\beta}\left(-p_{s_{0}}\right)^{k_{s_{0}}-m_{s_{0}}} \\
\ldots \prod_{s_{\beta-2}<s_{\beta-1}=\beta}^{\beta}\left((-1)^{\beta} p_{s_{0}} p_{s_{1}} \cdots p_{s_{\beta-1}}\right)^{k_{2} \beta_{-1}-m_{2} \beta_{-1}} .
\end{gathered}
$$

\section{Sums of Products}

Having developed the expressions for the Möbius Bernoulli numbers in the previous section, we will now use them in expressing the sums of products of the Möbius-Bernoulli power sums $\Psi_{k}(x, n)$.

Definition 10. We define sums of products of the Möbius Bernoulli power sums as $\Psi_{k}^{N}(x, n):=\sum_{\sum_{i=1}^{N} k_{i}=k}\left(\begin{array}{c}k \\ k_{1} \cdots k_{N}\end{array}\right) \Psi_{k_{1}}$ $(x, n) \cdots \Psi_{k_{N}}(x, n)$ for nonnegative integers $k$ and $N$ which are described by the generating function

$$
\left(\sum_{d \mid n} \mu(d) \frac{e^{(1+(x / d)) t d}-e^{t d}}{e^{t d}-1}\right)^{N}=\sum_{k=0}^{\infty} \Psi_{k}^{N}(x, n) \frac{t^{k}}{k !} .
$$

The next result evaluates the sums of products $\Psi_{k}^{N}(x, n)$.

Theorem 11. For a positive integer $N$ and nonnegative integer $k$,

$$
\begin{aligned}
& \Psi_{k}^{N}(x, n) \\
& \quad=\frac{k ! N !}{(k+N) !} \sum_{j=0}^{k}\left(\begin{array}{c}
k+N \\
j
\end{array}\right) M_{j}^{N}(n) S(k+N-j, N) x^{k+N-j}
\end{aligned}
$$

for all $n=2,3, \ldots$ where $S(\ell, m)$ are the Stirling numbers of second kind.
Proof. Observe from the generating function for $\Psi_{k}^{N}(x)$ that

$$
\begin{aligned}
\left(\sum_{d \mid n} \mu\right. & \left.(d) \frac{e^{(1+(x / d)) t d}-e^{t d}}{e^{t d}-1}\right)^{N} \\
& =\left(\sum_{d \mid n} \mu(d) \frac{t e^{t d}}{e^{t d}-1}\right)^{N}\left(\frac{e^{x t}-1}{t}\right)^{N} \\
& =\left(t \sum_{d \mid n} \mu(d)+\sum_{d \mid n} \frac{t \mu(d)}{e^{t d}-1}\right)^{N}\left(\frac{e^{x t}-1}{t}\right)^{N} \\
& =\left(t \delta_{1 n}+\sum_{d \mid n} \frac{t \mu(d)}{e^{t d}-1}\right)^{N}\left(\frac{e^{x t}-1}{t}\right)^{N} \\
& =\sum_{m=0}^{N}\left(\begin{array}{l}
N \\
m
\end{array}\right) t^{N-m} \delta_{1 n}^{N-m}\left(\sum_{d \mid n} \frac{t \mu(d)}{e^{t d}-1}\right)^{m}\left(\frac{e^{x t}-1}{t}\right)^{N}
\end{aligned}
$$

which on further simplification gives

$$
\begin{aligned}
& \sum_{k=0}^{\infty} \Psi_{k}^{N}(x, n) \frac{t^{k}}{k !} \\
&=\sum_{k=0}^{\infty} \sum_{m=0}^{N}\left(\begin{array}{l}
N \\
m
\end{array}\right) \sum_{j=0}^{k}\left(\begin{array}{l}
k \\
j
\end{array}\right) \delta_{1 n} M_{k-j}^{m}(n) \\
& \times \frac{j ! N !}{(j+N) !} S(j+N, N) x^{j+N} \frac{t^{k}}{k !},
\end{aligned}
$$

where we have used the identity $\left(\left(e^{x t}-1\right) / t\right)^{N}=\sum_{j=0}^{\infty}((k ! N ! /$ $\left.(j+N) !) S(j+N, N) x^{j+N}\left(t^{j} / j !\right)\right)$. On comparing like powers of $t$ in (30) we obtain

$$
\begin{gathered}
\Psi_{k}^{N}(x, n) \\
=\sum_{m=0}^{N}\left(\begin{array}{l}
N \\
m
\end{array}\right) \sum_{j=0}^{k}\left(\begin{array}{l}
k \\
j
\end{array}\right)\left(\delta_{1 n}\right)^{N-m} M_{k-j}^{m}(n) \frac{j ! N !}{(j+N) !} \\
\times S(j+N, N) x^{j+N}
\end{gathered}
$$

for all $k=0,1, \ldots ; n, N \in \mathbb{Z}^{+}$, where we define $\delta_{1 n}^{0}:=1$ for all $n \in \mathbb{Z}^{+}$. The result follows now.

Note that, from Theorem 11, we recover for $N=1$,

$$
\Psi_{k}^{1}(x, n)=\frac{1}{k+1} \sum_{j=0}^{k}\left(\begin{array}{c}
k+1 \\
j
\end{array}\right) x^{k+1-j} B_{j} \prod_{p \mid n}\left(1-p^{j-1}\right),
$$

where we have used $M_{j}^{1}(n)=B_{j} \prod_{p \mid n}\left(1-p^{j-1}\right)$ and $S(k+1-$ $j, 1)=1$ for all $j=0,1, \ldots, k$.

\section{Conflict of Interests}

The author declares that there is no conflict of interests regarding the publication of this paper. 


\section{Acknowledgments}

Many suggestions regarding presentation of the paper by Professor László Tóth are gratefully acknowledged. The author is also thankful to the anonymous referees for their suggestions.

\section{References}

[1] J. Singh, "Defining power sums of $n$ and $\varphi(n)$ integers," International Journal of Number Theory, vol. 5, no. 1, pp. 41-53, 2009.

[2] H. W. Gould, "Explicit formulas for Bernoulli numbers," The American Mathematical Monthly, vol. 79, no. 1, pp. 44-51, 1972.

[3] E. Alkan, "Distribution of averages of Ramanujan sums," The Ramanujan Journal, vol. 29, pp. 385-408, 2012.

[4] H. M. Srivastava and P. G. Todorov, "An explicit formula for the generalized Bernoulli polynomials," Journal of Mathematical Analysis and Applications, vol. 130, no. 2, pp. 509-513, 1988.

[5] W. P. Johnson, “The curious history of Faà di Bruno's formula," American Mathematical Monthly, vol. 109, no. 3, pp. 217-234, 2002. 


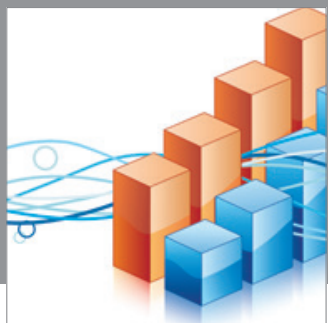

Advances in

Operations Research

mansans

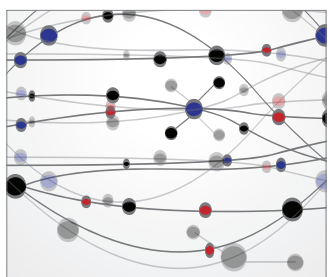

The Scientific World Journal
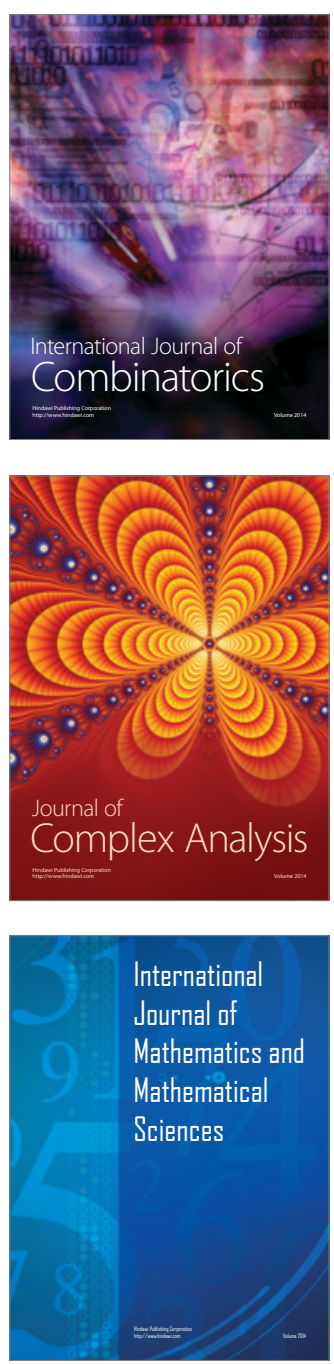
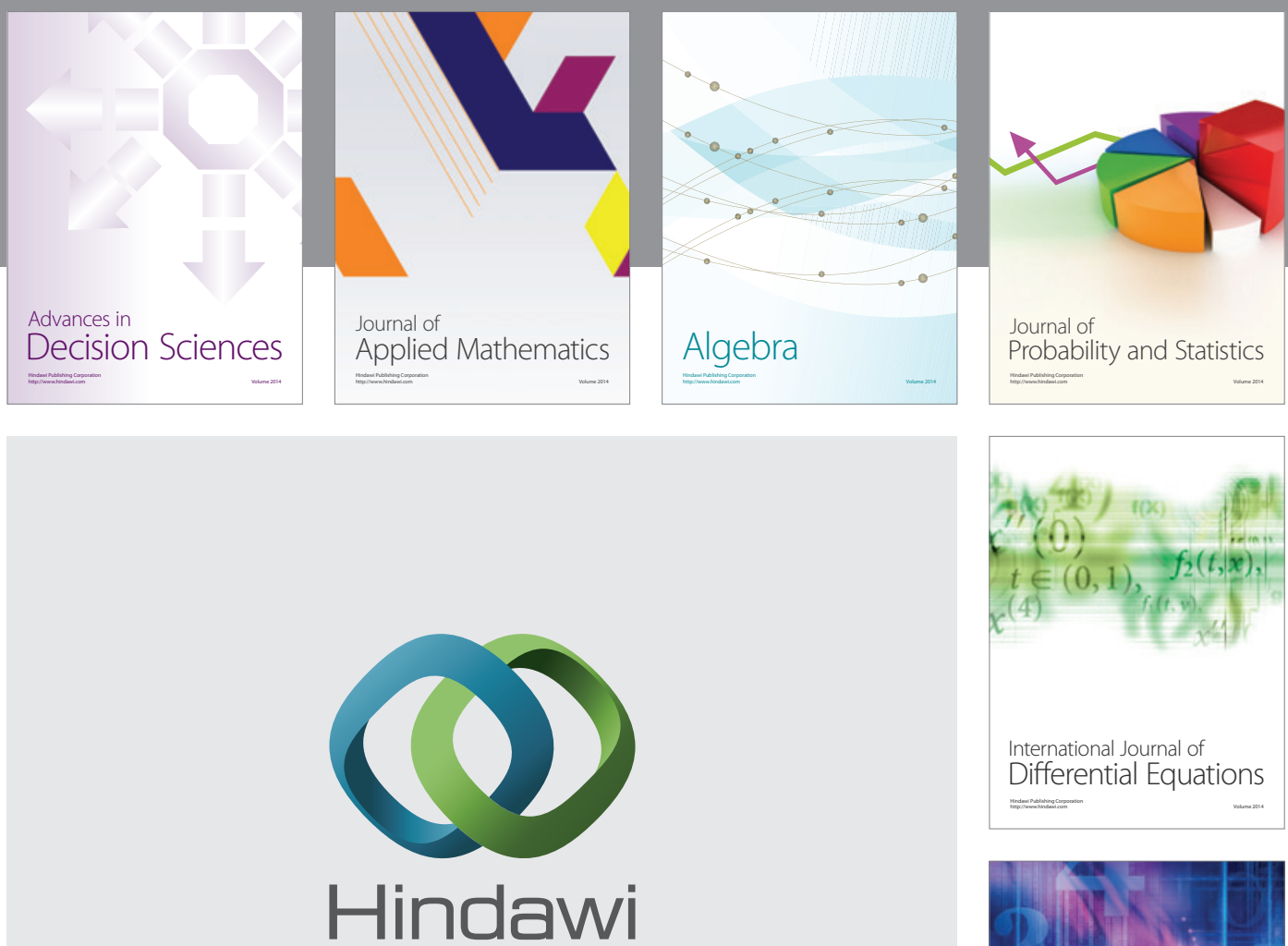

Submit your manuscripts at http://www.hindawi.com
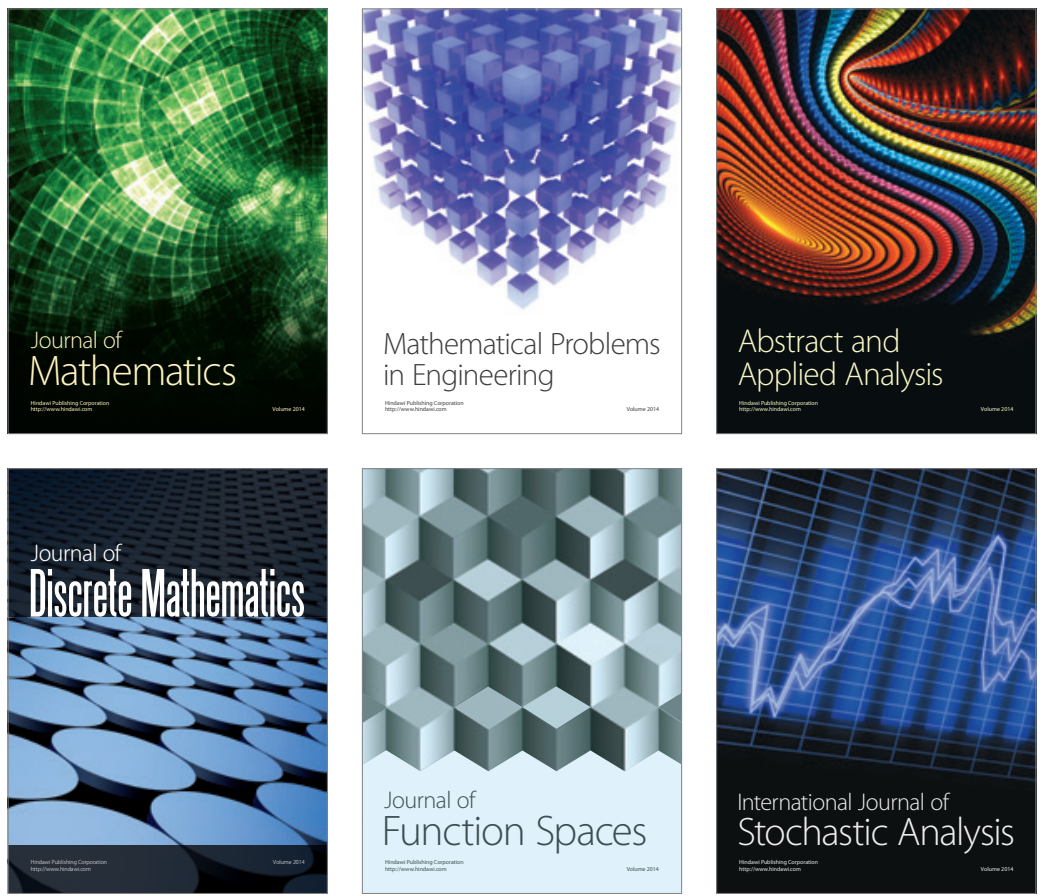

Journal of

Function Spaces

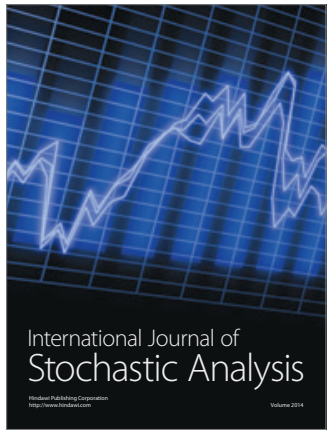

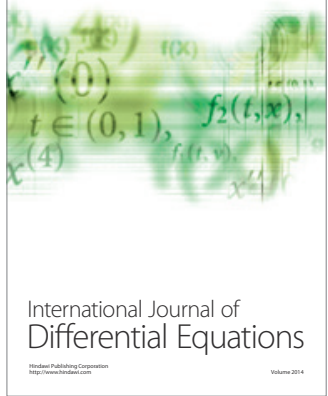
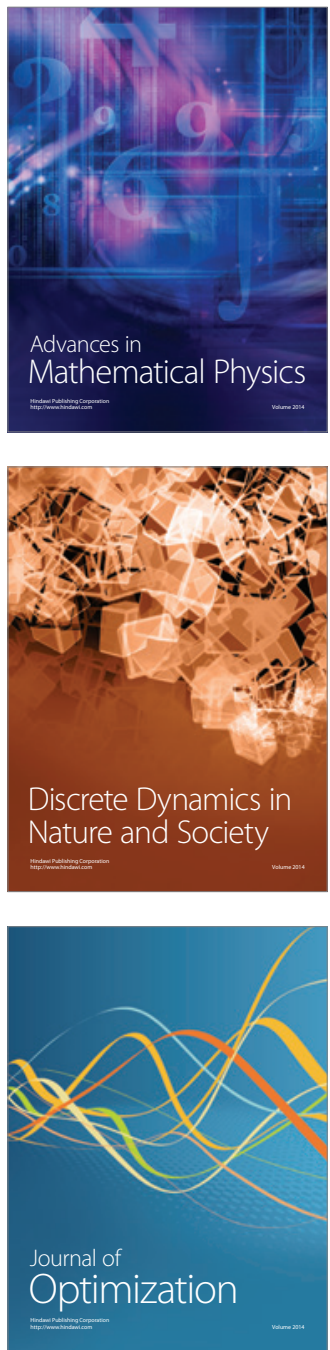